Research Paper

\title{
TheAssociation Between Inflammatory Biomarkers and Vitamin D Level With the Evolution and Severity of Stroke
}

\author{
Anahid Safari ${ }^{1}$ (D), Nima Fadakar ${ }^{2}$ (D), Afshin Borhani-Haghighi ${ }^{\star}$ (D) \\ 1. Stem Cells Technology Research Center, Shiraz University of Medical Sciences, Shiraz, Iran \\ 2. Clinical Neurology Research Center, Shiraz University of Medical Sciences, Shiraz, Iran.
}

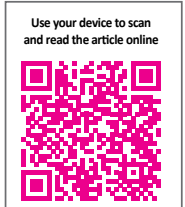

dtation Safari, A., Fadakar, N., and Borhani-Haghighi, A. (2022). The Association Between Inflammatory Biomarkers and Vitamin D Level With the Evolution and Severity of Stroke. Basic and Clinical Neuroscience, 13(6), 807-814. http://dx.doi. $\operatorname{org} / 10.32598 /$ bcn.2021.1971.1

http://dx.doi.org/10.32598/ben.2021.1971.1

Article info:

Received: 06 Aug 2019

First Revision: 14 Dec 2019

Accepted: 10 Feb 2020

Available Online: 01 Nov 2022

Keywords:

Ischemic stroke,

Cerebrovascular accident,

Vitamin D, High sensitive

C-reactive protein, Serum

Amyloid A, Osteopontin

\section{AB S T RA C T}

Introduction: Vitamin D deficiency has been linked to the evolution of ischemic stroke, but the data regarding the association between stroke severity and vitamin $\mathrm{D}$ level is scarce.

Methods: Patients with first-ever ischemic stroke in the middle cerebral artery territory, within seven days after the stroke, were recruited. The control group included age- and gendermatched individuals. We compared 25-OH vitamin D (vitamin D), high sensitive C-reactive protein (hsCRP), serum amyloid A (SAA), and osteopontin levels between stroke patients and the control group. The association between stroke severity according to the National Institutes of Health Stroke Scale (NIHSS) and the Alberta stroke program early CT score (ASPECTS) and levels of vitamin D and inflammatory biomarkers were also studied.

Results: There was an association between hypertension $(\mathrm{P}=0.035)$, diabetes mellitus ( $\mathrm{P}=0.043)$, smoking $(\mathrm{P}=0.016)$, history of ischemic heart disease $(\mathrm{P}=0.002)$, higher SAA $(\mathrm{P}<0.001)$, higher hsCRP $(\mathrm{P}<0.001)$, and lower vitamin $\mathrm{D}$ levels $(\mathrm{P}=0.002)$ and stroke evolution in a case-control study. Meanwhile, in stroke patients, its severity was associated with higher SAA $(\mathrm{P}=0.04)$ and hsCRP $(\mathrm{P}=0.001)$, and lower vitamin $\mathrm{D}$ levels ( $\mathrm{P}=0.043$ ) according to clinical scale (higher admission NIHSS). According to the ASPECT score, higher SAA $(\mathrm{P}=0.017)$ and hsCRP $(\mathrm{P}=0.007)$, but not lower vitamin $\mathrm{D}$ levels, were associated with more infarct areas $(\mathrm{P}=0.149)$.

Conclusion: Vitamin D may play a role in both the evolution and severity of stroke.

\footnotetext{
* Corresponding Author: Afshin Borhani-Haghighi, MD.

Address: Clinical Neurology Research Center, Shiraz University of Medical Sciences, Shiraz, Iran

Tel: +98 (71) 36272287

E-mail: neuro.ab@gmail.com
} 


\section{Highlights}

- Low Vitamin D was associated with stroke evolution.

- High sensitive CRP and serum amyloid A were associated with stroke evolution.

- Stroke severity was associated with increased inflammatory biomarkers, and lower vitamin D.

- Vitamin D deficiency may contribute in evolution of stroke through inflammatory mechanisms.

\section{Plain Language Summary}

Plain Language summary: As stroke is one of the most common causes of death and disability all over the world, finding contributing factors in its evolution is important. Some previous studies showed the association between Vitamin D deficiency and the formation of stroke. In this study, we showed that both low vitamin D levels and increased inflammatory markers were associated with a higher risk of stroke. These inflammatory markers are some biochemical substances that increase during the inflammatory cascade. In addition, more severe stroke patients had lower levels of vitamin D and higher inflammatory markers. We concluded that insufficient dietary vitamin D may result in stroke through the induction of an inflammatory mechanism. As a practical therapeutic implication, vitamin D supplements for vulnerable patients may decrease the risk of stroke.

\section{Introduction}

troke is among the most common causes of mortality and morbidity globally (Borhani-Haghighi et al., 2013); however, our armamentarium for its treatment is limited (Shahtaheri, et al., 2012). Numerous pieces of evidence have revealed the crucial role of inflammation in the pathogenesis of acute ischemic stroke. Hence, inflammatory biomarkers might predict the functional outcome of the stroke (Safari, et al., 2016).

C-reactive protein (CRP) or Pentraxin1(Ptx1), is made by liver cells in response to interleukin-1 (IL-1), IL-6, and tumor necrotic factor-alpha (TNF- $\alpha$ ). Highsensitivity CRP (hsCRP) picks up even low CRP (Mohebbi et al., 2012).

Serum amyloid A (SAA) is also an acute-phase reactant that increases within hours after an inflammatory reaction starts. Some studies have suggested the superiority of SAA over erythrocyte sedimentation rate (ESR) and CRP in quantifying inflammatory reactions (Cunnane et al., 2000).

Osteopontin (OPN) is a secreted extracellular phosphoprotein, which plays a pivotal role in the normal mineralization of bone as well as up-regulating calcified plaques of the vessels. In addition, OPN has immunomodulatory activities via chemotaxis, cell attachment, cytokine production, and anti-apoptosis mechanism (Wang \& Denhardt, 2008).

Low 25-hydroxyvitamin D (25-OH vitamin D) levels have been reported to be associated with obesity, diabetes mellitus (DM), dyslipidemia, and hypertension, which are the major risk factors in ischemic stroke (Muscogiuri et al., 2017).

Even though there is increasing evidence regarding the association between vitamin $\mathrm{D}$ deficiency and an increased risk of stroke "evolution" (Zhou et al., 2018), the association between vitamin D level and the severity of the stroke and its inflammatory aspects is still unclear. The severity of stroke is assessed by both clinical measures, such as the National Institutes of Health Stroke Scale (NIHSS), and radiologic measures, like the Alberta Stroke Program Early CT Score (ASPECTS), the two available criteria for assessing stroke severity.

There are a few studies that have investigated the association between NIHSS as a stroke severity measure and vitamin D level at the time of admission (Daubail et al., 2013; Park et al., 2015; Tu, Zhao, Xu, \& Chen, 2014; Wang, Ji, Tong, \& Zhang, 2014; Wei \& Kuang, 2018; Zhang, Wang, Zhong, Liao, \& Lu, 2018). However, to the best of our knowledge, no study has investigated the association between radiological severity scales, such as ASPECTS, and vitamin D levels. In addition, the acute phase reactants were not analyzed in the aforementioned studies. 
We compared serum 25-OH vitamin D (vitamin D), hsCRP, SAA, and osteopontin levels between patients with middle cerebral artery (MCA) infarction and agegender-matched normal individuals. We also investigated the association between stroke severity according to the NIHSS and the ASPECTS, vitamin D levels, and inflammatory biomarkers. We hypothesized that vitamin $D$ deficiency might induce a larger infarct area according to ASPECTS through modulation of immunologic factors, such as hsCRP, SAA, and osteopontin.

\section{Materials and Methods}

This is a prospective cross-sectional study conducted in Namazi hospital, a high-volume center for stroke in southwestern Iran, affiliated with the Shiraz University of Medical Sciences from June 2017 to March 2018.

\section{Inclusion criteria}

Patients with first-ever acute ischemic stroke in the middle cerebral artery (MCA) territory within seven days before recruitment were enrolled. According to the Recognition of Stroke in the Emergency Room (ROSIER) scale (Nor et al., 2005), ischemic stroke was defined as an acute focal neurological deficit, presented for at least 24 hours, and confirmed by a brain CT or an MRI. Other inclusion criteria were the age of more than 18 years and providing written informed consent.

\section{The exclusion criteria}

Patients with primary intracranial hemorrhage were excluded. Patients with recurrent ischemic stroke and ischemic stroke that lasted more than seven days were also not included.

Patients with lacunar stroke [Causative Classification System for Ischemic Stroke (CSS type III)] (Ay et al., 2005) were also excluded.

Patients with cervico-cephalic arterial dissection, vasculitides, cerebral venous sinus thrombosis, hypoperfusion syndromes, iatrogenic stroke, vasospasm after subarachnoid hemorrhage, meningitis, hematologic causes of stroke, Moyamoya disease, and reversible vasoconstriction syndrome were also excluded.

Conditions that could affect vitamin D, hsCRP, SAA, and osteopontin levels, such as atrial fibrillation (Cheng, et al., 2012; Thompson, et al., 2015), fever or any other sign of infection, any previously proven neoplastic (Garland et al., 2006; Moshkovskii, 2012), rheumatologic or inflammatory diseases (Hwang et al., 2016), renal failure (Dieter et al., 2016; Tonelli, et al., 2005), acute or chronic liver failure (Maury, Teppo, \& Salaspuro, 1983), respiratory failure (Bozinovski et al., 2008), recent surgery (Habib, et al., 2009), thyroid (Reza,et al., 2013), and parathyroid diseases (Maser, et al., 2018), Alzheimer's disease (Chung et al., 2000), Parkinson's disease and any history of osteoporosis (Abdu-Allah, et al., 2015) or its related risk factors were also excluded. Patients with excessive alcohol intake were not recruited as well (Hillborn, 1998).

Patients who received vitamin D or calcium supplements, anticoagulants, glucocorticoids, other anti-inflammatory drugs, calcitonin, parathyroid hormone, and osteoporosis-related medications were also excluded.

\section{Control group}

The control group included age-and-gender-matched individuals with neither any history of ischemic stroke/ transient ischemic attack (TIA) nor any of the abovementioned exclusion criteria.

\section{Sample size and variables}

The sample size was calculated based on $\alpha=0.05$, $\beta=0.80$, and the effect size of 0.6 by G Power Software. Body mass index (BMI) was measured in the case and control groups. Major cerebrovascular risk factors, including current or previous cigarette smoking habits, hypertension, diabetes mellitus (DM), dyslipidemia, and a history of ischemic heart disease (IHD), were investigated for all participants.

\section{Laboratory investigations}

Ten cc blood was obtained from patients 2-7 days after the stroke, and from the controls. The sera were banked at $-80^{\circ} \mathrm{C}$ and then defrosted. Calcium, phosphor, alkaline phosphatase (ALP), fasting blood sugar (FBS), blood urea nitrogen $(\mathrm{BUN})$, and creatinine $(\mathrm{Cr})$ were measured by standard techniques. $25-\mathrm{OH}$ vitamin $\mathrm{D}$ was quantified by the enzyme-linked immunosorbent assay (ELISA) method. The details were mentioned in our previous publication (Safari et al., 2019)

\section{Study ethics}

The study was approved by the ethics committee of Shiraz University of Medical Sciences (No\#1396-S104). Written informed consent was taken from both patients and controls. If the patients were confused or unconscious, consent was taken from their next of kin. 


\section{Statistical analysis}

Statistical analyses were conducted using SPSS version 20. For variables with a normal distribution, the results were presented with a mean and $95 \%$ confidence interval (CI). The Chi-square test and independent $t$-test were used to test the differences. The logistic regression model was utilized to determine the effects of hypertension, DM, smoking, IHD, serum levels of hsCRP, SAA, osteopontin, and vitamin D on stroke evolution. Pearson correlation coefficient was used to find the association between stroke severity scales (NIHSS and ASPECT) and serum concentration of biomarkers (hsCRP, SAA, osteopontin, and vitamin D). The significance level of 0.05 was deemed appropriate.

\section{Results}

Eighty-nine patients with acute ischemic stroke were initially recruited. After applying all the inclusion and exclusion criteria, 46 patients with the first-ever attack of MCA infarction were selected. The 46 age-and-gendermatched healthy subjects were recruited as the control population. Table 1 shows demographic variables, stroke risk factors, calcium, vitamin D level, hsCRP, SAA, and osteopontin levels.

On evaluating the risk factors and serum biomarkers with stroke evolution, univariate analysis revealed the association of DM, hypertension, smoking, and the history of IHD, in addition to higher SAA, higher hsCRP, and lower vitamin D levels with the evolution of stroke (Table 2).

In stroke patients, significant reverse associations between vitamin $\mathrm{D}$ and SAA levels $(\mathrm{R}=-0.241, \mathrm{P}=0.025)$, and vitamin $\mathrm{D}$ and hsCRP levels $(\mathrm{R}=-0.267, \mathrm{P}=0.011)$ were detected. However, there was no significant association between vitamin $\mathrm{D}$ and serum osteopontin levels $(\mathrm{R}=0.051, \mathrm{P}=0.642)$.

In stroke patients, the mean and $95 \% \mathrm{CI}$ of admission NIHSS score were 12.97 and 10.65-15.35, respectively. In addition, the mean and $95 \%$ CI of their ASPECT score were 6.24 and 5.57-6.83, respectively.

There was a significant reverse association between NIHSS score and vitamin $\mathrm{D}$ level $(\mathrm{R}=-0.306, \mathrm{P}=0.043)$. Meanwhile, a significant association between NIHSS

Table 1. Comparison between demographic characteristics, risk factors, and laboratory results in patients and healthy subjects

\begin{tabular}{|c|c|c|c|}
\hline \multirow{2}{*}{ Variables } & \multicolumn{2}{|c|}{ Mean $(95 \% \mathrm{Cl}) /$ No. (\%) } & \multirow{2}{*}{ P-Value } \\
\hline & Case $(n=46)$ & Control $(n=46)$ & \\
\hline Age & $64.22(59.88-68.30)$ & $66.72(63.78-69.65)$ & 0.33 \\
\hline Sex & $23(50.0)$ & $23(50.0)$ & - \\
\hline Body mass index $\left(\mathrm{kg} / \mathrm{m}^{2}\right)$ & $25.55(24.52-27.11)$ & 26.85(24.39-29.86) & 0.34 \\
\hline Hypertension & $25(54.3)$ & $15(32.6)$ & 0.035 \\
\hline Hyperlipidemia & $13(28.3)$ & $11(24.4)$ & 0.68 \\
\hline Diabetes Mellitus & $14(30.4)$ & $6(13.0)$ & 0.043 \\
\hline Smoking & $13(28.3)$ & $4(8.7)$ & 0.016 \\
\hline Ischemic heart disease & $15(32.6)$ & $3(6.5)$ & 0.002 \\
\hline Received Tissue-Plasminogen activator (tPA) & $5(10.9)$ & $0(0)$ & - \\
\hline Serum Amyloid A (ng/mL) & $808.39(214-340.36)$ & 233.07(130.83-398.48) & $<0.001$ \\
\hline $\mathrm{hsCRP}(\mathrm{ng} / \mathrm{mL})$ & 7054.70(6461.68-7871.40) & $1833.62(1194.45-4067.38)$ & $<0.001$ \\
\hline Osteopontin (ng/mL) & $15.34(10.90-23.76)$ & $16.92(13.34-45.70)$ & 0.70 \\
\hline $25-\mathrm{OH}$ vitamin $\mathrm{D}(\mathrm{ng} / \mathrm{mL})$ & $22.40(18.83-25.86)$ & $32.07(20.13-39.12)$ & 0.002 \\
\hline
\end{tabular}

$\mathrm{CI}=$ Confidence interval. 
Table 2. Univariate analysis of various risk factors with stroke evolution

\begin{tabular}{cccc}
\hline Variables & OR & Cl & P \\
\hline Hypertension & 2.46 & $1.05-5.73$ & 0.037 \\
\hline Diabetes Mellitus & 2.91 & $1.007-8.44$ & 0.048 \\
Smoking & 0.24 & $0.072-0.81$ & 0.021 \\
\hline Ischemic heart disease & 6.93 & $1.84-26.03$ & 0.004 \\
\hline Serum Amyloid A & 1.006 & $1.004-1.009$ & $<0.001$ \\
hsCRP & 1.001 & $1.001-1.002$ & $<0.001$ \\
\hline Osteopontin & 0.99 & $0.97-1.001$ & 0.70 \\
\hline 25-OH vitamin D & 0.94 & $0.91-0.98$ & 0.005 \\
\hline
\end{tabular}

OR= Odds ratio; $\mathrm{CI}=95 \%$ Confidence interval.

score and hsCRP $(\mathrm{R}=0.482, \mathrm{P}=0.001)$, as well as SAA levels $(\mathrm{R}=0.315, \mathrm{P}=0.04)$ were found. However, the reverse association between NIHSS score and osteopontin level was not significant $(\mathrm{R}=-0.091, \mathrm{P}=0.56)$.

When stroke severity was evaluated according to ASPECT, there was a significant reverse association between ASPECT score and hsCRP and SAA levels $(\mathrm{R}=$ $-0.401, \mathrm{P}=0.007$ and $\mathrm{R}=-0.361, \mathrm{P}=0.017)$. However, no significant association between ASPECT score and vitamin $\mathrm{D}$ and osteopontin levels $(\mathrm{R}=0.221$ and -0.071 , $\mathrm{P}=0.149$ and 0.651 ) was seen.

\section{Discussion}

The current study showed the association of hypertension, DM, smoking, history of ischemic heart disease, higher SAA, higher hsCRP, and lower vitamin D levels with stroke evolution in a case-controlled study. Meanwhile, in stroke patients, its severity was associated with higher SAA, higher hsCRP, and lower vitamin D according to the clinical scale (higher admission NIHSS). According to the ASPECT score, higher SAA and hsCRP, but not lower vitamin D levels, were associated with more infarct areas. NIHSS and ASPECT scores were not significantly associated with osteopontin levels.

Our results are consistent with previous studies, which showed the association between vitamin D deficiency and stroke evolution (Zhang et al., 2017). The anti-calcific protective effect of vitamin $\mathrm{D}$ in the process of atherogenesis might prevent the evolution of atherothrombotic ischemic stroke (Mozos \& Marginean, 2015).
Meanwhile, vitamin D deficiency was associated with higher NIHSS in all (Afshari, Amani, Soltani, Haghighizadeh, \& Afsharmanesh, 2015; Daubail et al., 2013; Park et al., 2015; Tu et al., 2014; Wang et al., 2014), as well as subgroups of acute ischemic stroke patients (Zhou et al., 2018). The anti-inflammatory effect of vitamin D might play a role in stroke severity. There are both animal and human studies supporting this hypothesis. Balden et al. showed larger infarct volumes and more severe behavioral disturbances in rats fed with a vitamin D-deficient diet compared to controls. They hypothesized the activation of inflammatory markers and suppression of inherent neuroprotective agents, such as Insulin-growth factor-1. Interestingly, vitamin D injection in the acute stage did not improve infarct volume or behavioral disturbance (Balden, Selvamani, \& Sohrabji, 2012). Wang et al. stated that serum vitamin D levels were adversely associated with serum levels of IL-6 and hsCRP in stroke patients (Wang, et al., 2018) .

As far as we know, few studies have studied the link between ASPECT score with vitamin D levels, hsCRP, SAA, and osteopontin. The current study failed to show the association between ASPECT score and vitamin D or osteopontin levels. However, it revealed a significant association between lower ASPECT scores (i.e. larger infarct areas) and higher levels of hsCRP and SAA.

The fundamental limitation of the current study was the small sample size. We deleted all the confounding factors affecting vitamin D, hsCRP, SAA, and osteopontin levels to increase the reliability of the results. Finding a pure group of MCA infarction with the exclusion of all confounding factors was the primary obstacle. 
In conclusion, the present study showed vitamin D's speculative role in both the evolution and severity of stroke. The protective effects of vitamin D might be due to its immune-modulatory activities. More extensive studies are warranted to investigate the benefits of vitamin $\mathrm{D}$ supplementation in preventing and treating stroke patients.

\section{Ethical Considerations}

\section{Compliance with ethical guidelines}

The study was approved by the ethics committee of Shiraz University of Medical Sciences (No.: \#1396S104). Written informed consent was taken from both patients and controls. If the patients were confused or unconscious, consent was taken from their next of kin.

\section{Funding}

This study is in partial fulfillment of the thesis project for the specialty of neurology degree by Dr. Nima Fadakar. This study is financially supported by the Office of Vice Chancellor for Research of Shiraz University of Medical Sciences (Grant No.: \#13990).

\section{Authors' contributions}

All authors equally contributed to preparing this article.

\section{Conflict of interest}

The authors declare no conflict of interest.

\section{Acknowledgments}

We thank Heydari and Neydavoodi for statistical analysis; Zafarmnad for data collection; and H. Argasi at the Research Consultation Center (RCC) of Shiraz University of Medical Sciences for literal editing.

\section{References}

Abdu-Allah, A. M., Tarhouny, S. A., \& Baghdadi, H. H. (2015). Serum amyloid a gene polymorphism and its association with lipid profile in Saudi females with osteoporosis. Pakistan Journal of Medical Sciences, 31(5), 1124-1129. [DOI:10.12669/pjms.315.7981] [PMID] [PMCID]

Afshari, L., Amani, R., Soltani, F., Haghighizadeh, M. H., \& Afsharmanesh, M. R. (2015). The relation between serum Vitamin D levels and body antioxidant status in ischemic stroke patients: A case-control study. Advanced Biomedical Research, 4, 213. [PMID]
Ay, H., Furie, K. L., Singhal, A., Smith, W. S., Sorensen, A G., \& Koroshetz, W. J. (2005). An evidence-based causative classification system for acute ischemic stroke. Annals of Neurology, 58(5), 688-697. [DOI:10.1002/ana.20617] [PMID]

Balden, R., Selvamani, A., \& Sohrabji, F. (2012). Vitamin D deficiency exacerbates experimental stroke injury and dysregulates ischemia-induced inflammation in adult rats. Endocrinology, 153(5), 2420-2435. [DOI:10.1210/en.2011-1783] [PMID] [PMCID]

Borhani-Haghighi, A., Safari, R., Heydari, S. T., Soleimani, F., Sharifian, M., \& Kashkuli, S. Y., et al. (2013). Hospital mortality associated with stroke in southern Iran. Iranian Journal of Medical Sciences, 38(4), 314. [DOI:10.1016/j.jns.2013.07.750]

Bozinovski, S., Hutchinson, A., Thompson, M., Macgregor, L., Black, J., \& Giannakis, E., et al. (2008). Serum amyloid a is a biomarker of acute exacerbations of chronic obstructive pulmonary disease. American Journal of Respiratory and Critical Care Medicine, 177(3), 269-278. [PMID]

Cheng, T., Wang, X. F., Hou, Y. T., \& Zhang, L. (2012). Correlation between atrial fibrillation, serum amyloid protein A and other inflammatory cytokines. Molecular Medicine Reports, 6(3), 581-584. [DOI:10.3892/mmr.2012.934] [PMID]

Chung, T. F., Sipe, J. D., McKee, A., Fine, R. E., Schreiber, B. M., \& Liang, J. S., et al. (2000). Serum amyloid A in Alzheimer's disease brain is predominantly localized to myelin sheaths and axonal membrane. Amyloid, 7(2), 105-110. [DOI:10.3109/13506120009146246] [PMID]

Cunnane, G., Grehan, S., Geoghegan, S., McCormack, C., Shields, D., \& Whitehead, A. S., et al. (2000). Serum amyloid $\mathrm{A}$ in the assessment of early inflammatory arthritis. The Journal of Rheumatology, 27(1), 58-63. [PMID]

Daubail, B., Jacquin, A., Guilland, J. C., Hervieu, M., Osseby, G. V., \& Rouaud, O., et al. (2013). Serum 25-hydroxyvitamin D predicts severity and prognosis in stroke patients. European Journal of Neurology, 20(1), 57-61. [DOI:10.1111/ j.1468-1331.2012.03758.x] [PMID]

Dieter, B. P., McPherson, S. M., Afkarian, M., de Boer, I. H., Mehrotra, R., \& Short, R., et al. (2016). Serum amyloid a and risk of death and end-stage renal disease in diabetic kidney disease. Journal of Diabetes and Its Complications, 30(8), 14671472. [PMID] [PMCID]

Garland, C. F., Garland, F. C., Gorham, E. D., Lipkin, M., Newmark, H., \& Mohr, S. B., et al. (2006). The role of vitamin D in cancer prevention. American Journal of Public Health, 96(2) 252-261. [DOI:10.2105/AJPH.2004.045260] [PMID] [PMCID]

Habib, P., Scrocco, J. D., Terek, M., Vanek, V., \& Mikolich, J. R. (2009). Effects of bariatric surgery on inflammatory functional and structural markers of coronary atherosclerosis. The American Journal of Cardiology, 104(9), 1251-1255. [DOI:10.1016/j.amjcard.2009.06.042] [PMID]

Hillborn, M. (1998). Alcohol consumption and stroke: Benefits and risks. Alcoholism: Clinical and Experimental Research 22(S7), 352s-358s. [DOI:10.1111/j.1530-0277.1998.tb04390.x]

Hwang, Y. G., Balasubramani, G. K., Metes, I. D., Levesque, M. C., Bridges, S. L., Jr, \& Moreland, L. W. (2016). Differential response of serum amyloid A to different therapies in early rheumatoid arthritis and its potential value as a disease activity biomarker. Arthritis Research \& Therapy, 18(1), 108. [DOI:10.1186/s13075-016-1009-y] [PMID] [PMCID] 
Maser, R. E., Lenhard, M. J., Pohlig, R. T., Balagopal, P. B., \& Abdel-Misih, R. (2018). Effect of parathyroidectomy on osteopontin and undercarboxylated osteocalcin in patients with primary hyperparathyroidism. Endocrine Research, 43(1), 21-28. [PMID] [PMCID]

Maury, C., Teppo, A. M., \& Salaspuro, M. P. (1983). Amyloid A fibril degrading activity in serum in liver disease-relation to serum acute phase and other protein levels. Clinica Chimica Acta, 131(1-2), 29-37. [DOI:10.1016/0009-8981(83)90349-2]

Mohebbi, S., Ghabaee, M., Ghaffarpour, M., Meisami, A. P., Siah, R. S., \& Mirkala, M. R., et al. (2012). Predictive role of high sensitive C-reactive protein in early onset mortality after ischemic stroke. Iranian Journal of Neurology, 11(4), 135-139. [PMID]

Moshkovskii, S. (2012). Why do cancer cells produce serum amyloid A acute-phase protein? Biochemistry (Moscow), 77(4), 339-341. [PMID]

Mozos, I., \& Marginean, O. (2015). Links between vitamin D deficiency and cardiovascular diseases. BioMed Research International, 2015, 109275. [DOI:10.1155/2015/109275] [PMID] [PMCID]

Muscogiuri, G., Annweiler, C., Duval, G., Karras, S., Tirabassi, G., \& Salvio, G., et al. (2017). Vitamin D and cardiovascular disease: From atherosclerosis to myocardial infarction and stroke. International Journal of Cardiology, 230, 577-584. [DOI:10.1016/j.ijcard.2016.12.053] [PMID]

Nor, A. M., Davis, J., Sen, B., Shipsey, D., Louw, S. J., \& Dyker, A. G., et al. (2005). The Recognition of Stroke in the Emergency Room (ROSIER) scale: Development and validation of a stroke recognition instrument. The Lancet Neurology, 4(11), 727-734. [DOI:10.1016/S1474-4422(05)70201-5] [PMID]

Park, K. Y., Chung, P. W., Kim, Y. B., Moon, H. S., Suh, B. C., \& Won, Y. S., et al. (2015). Serum vitamin D status as a predictor of prognosis in patients with acute ischemic stroke. Cerebrovascular Diseases, 40(1-2), 73-80. [DOI:10.1159/000434691] [PMID]

Reza, S., Shaukat, A., Arain, T. M., Riaz, Q. S., \& Mahmud, M. (2013). Expression of osteopontin in patients with thyroid dysfunction. PloS One, 8(2), e56533. [DOI:10.1371/journal. pone.0056533] [PMID] [PMCID]

Safari, A., Borhani-Haghighi, A., Dianatpour, M., Heydari, S. T., Foroughinia, F., \& Ranjbar Omrani, G. (2019). Circulating serum amyloid A, hs-CRP and vitamin D levels in postmenopausal osteoporosis. Galen Medical Journal, 8, e1548. [PMID] [PMCID]

Safari, A., Safari, R., \& Borhani-Haghighi, A. (2016). Immunology of stroke. Galen Medical Journal, 5(Supp.1), 10-17. [Link]

Shahtaheri, R. A., Borhani Haghighi, A., Safari, A., \& Cruz-Flores, S. (2012). Recombinant tissue plasminogen activator (rtPA) and stroke unit for acute ischaemic stroke in developing countries, are they cost-effective? International Journal of Stroke, 7(7), E9. [DOI:10.1111/j.1747-4949.2012.00892.x] [PMID]

Thompson, J., Nitiahpapand, R., Bhatti, P., \& Kourliouros, A. (2015). Vitamin D deficiency and atrial fibrillation. International Journal of Cardiology, 184, 159-162. [DOI:10.1016/j. ijcard.2015.02.012] [PMID]
Tonelli, M., Sacks, F., Pfeffer, M., Jhangri, G. S., Curhan, G., \& Cholesterol and Recurrent Events (CARE) Trial Investigators (2005) Biomarkers of inflammation and progression of chronickidney disease. Kidney International, 68(1), 237-245. [PMID]

Tu, W. J., Zhao, S. J., Xu, D. J., \& Chen, H. (2014). Serum 25-hydroxyvitamin D predicts the short-term outcomes of Chinese patients with acute ischaemic stroke. Clinical Science, 126(5), 339-346. [PMID]

Wang, K. X., \& Denhardt, D. T. (2008). Osteopontin: Role in immune regulation and stress responses. Cytokine $\mathcal{E}$ Growth Factor Reviews, 19(5-6), 333-345. [DOI:10.1016/j.cytogfr.2008.08.001] [PMID]

Wang, Q., Zhu, Z., Liu, Y., Tu, X., \& He, J. (2018). Relationship between serum vitamin $\mathrm{D}$ levels and inflammatory markers in acute stroke patients. Brain and Behavior, 8(2), e00885. [DOI:10.1002/brb3.885]

Wang, Y., Ji, H., Tong, Y., \& Zhang, Z. B. (2014). Prognostic value of serum 25-hydroxyvitamin $D$ in patients with stroke. Neurochemical Research, 39(7), 1332-1337. [DOI:10.1007/ s11064-014-1316-0] [PMID]

Wei, Z. N., \& Kuang, J. G. (2018). Vitamin D deficiency in relation to the poor functional outcomes in nondiabetic patients with ischemic stroke. Bioscience Reports, 38(2), BSR20171509. [PMID] [PMCID]

Zhang, B., Wang, Y., Zhong, Y., Liao, S., \& Lu, Z. (2018). Serum 25-hydroxyvitamin $\mathrm{D}$ deficiency predicts poor outcome among acute ischemic stroke patients without hypertension. Neurochemistry International, 118, 91-95. [PMID]

Zhang, R., Li, B., Gao, X., Tian, R., Pan, Y., \& Jiang, Y., et al. (2017). Serum 25-hydroxyvitamin D and the risk of cardiovascular disease: Dose-response meta-analysis of prospective studies. The American Journal of Clinical Nutrition, 105(4), 810-819. [DOI:10.3945/ajcn.116.140392] [PMID]

Zhou, R., Wang, M., Huang, H., Li, W., Hu, Y., \& Wu, T. (2018). Lower vitamin $\mathrm{D}$ status is associated with an increased risk of ischemic stroke: A systematic review and meta-analysis. Nutrients, 10(3), 277. [PMID] [PMCID] 
This Page Intentionally Left Blank 